\title{
Physical Layer \\ Security Issues in \\ Massive MIMO and \\ GNSS
}

Ziya Gülgün 

Linköping Studies in Science and Technology

Licentiate Thesis, No. 1899

\title{
Physical Layer Security Issues in Massive MIMO and GNSS
}

\author{
Ziya Gülgün
}

\section{Eommunication
Sŷstems}

Division of Communication Systems Department of Electrical Engineering (ISY) Linköping University, 58183 Linköping, Sweden www.commsys.isy.liu.se

Linköping 2021 
This is a Swedish Licentiate Thesis.

The Licentiate degree comprises 120 ECTS credits of postgraduate studies.

(cc) EY-NC This work is licensed under a Creative Commons AttributionNonCommercial 4.0 International License.

https://creativecommons.org/licenses/by-nc/4.0/

Physical Layer Security Issues in Massive MIMO and GNSS

(c) 2021 Ziya Gülgün, unless otherwise stated.

ISBN 978-91-7929-698-8

ISSN 0280-7971

Printed in Sweden by LiU-Tryck, Linköping 2021 


\section{Abstract}

Wireless communication technology has evolved rapidly during the last 20 years. Nowadays, there are huge networks providing communication infrastructures to not only people but also to machines, such as unmanned air and ground vehicles, cars, household appliances and so on. There is no doubt that new wireless communication technologies must be developed, that support the data traffic in these emerging, large networks. While developing these technologies, it is also important to investigate the vulnerability of these technologies to different malicious attacks. In particular, spoofing and jamming attacks should be investigated and new countermeasure techniques should be developed. In this context, spoofing refers to the situation in which a receiver identifies falsified signals, that are transmitted by the spoofers, as legitimate or trustable signals. Jamming, on the other hand, refers to the transmission of radio signals that disrupt communications by decreasing the signal-to-interference-and-noise ratio (SINR) on the receiver side.

In this thesis, we analyze the effects of spoofing and jamming both on global navigation satellite system (GNSS) and on massive multiple-input multiple-output (MIMO) communications. GNSS is everywhere and used to provide location information. Massive MIMO is one of the cornerstone technologies in $5 \mathrm{G}$. We also propose countermeasure techniques to the studied spoofing and jamming attacks.

More specifically, in paper A we analyze the effects of distributed jammers on massive MIMO and answer the following questions: Is massive MIMO more robust to distributed jammers compared with previous generation's cellular networks? Which jamming attack strategies are the best from the jammer's perspective, and can the jamming power be spread over space to achieve more harmful attacks? In paper B, we propose a detector for GNSS receivers that is able to detect multiple spoofers without having any prior information about the attack strategy or the number of spoofers in the environment. 


\section{Populärvetenskaplig Sammanfattning}

Trådlösa kommunikationsteknologier har utvecklats snabbt, speciellt under de senaste 20 åren. Idag finns det mycket stora nätverk som består inte bara av människor utan också av maskiner, som t.ex. obemannade flygande och markbundna fordon, bilar, hushållsmaskiner och så vidare. Därför är det ingen tvekan om att nya trådlösa kommunikationsteknologier bör utvecklas, som stödjer datatrafiken i dessa stora nätverk. Dock, denna utveckling kräver också undersökning av sårbarheter hos dessa teknologier för skadliga attacker, som t.ex. spoofing och jamming, och nya tekniker för att motverka detta bör föreslås.

Spoofing utgör en situation där en mottagare identifierar falska signaler som skickats av spoofers som legitima eller pålitliga signaler.Jamming, å andra sidan, utgör transmission av radiosignaler som stör kommunikationer genom att minska signal-till-brus-förhållandet (SNR) på mottagarsidan.

I detta arbete analyserar vi effekten av spoofing och jamming på globala satellitsystem för navigation (GNSS) och massiv multipel-in-multipel-ut (MIMO), som är en av de starka teknologierna för 5G och föreslår motmedeltekniker. Specifikt föreslår vi en detektor för GNSS-mottagare som kan detektera flera spoofers utan att i förväg känna till attackstrategin och antalet spoofers i omgivningen. Ett annat bidrag i detta verk är att vi analyserar effekten på massiv MIMO av distribuerade jammers och besvarar följande frågor: Är massiv MIMO mer robust mot distribuerade jammers jämfört med tidigare generationer av cellulära nät? Vilken attackstrategi för jamming är bäst ur jammerns synvinkel, och behöver effekten för jammingen spridas geografiskt för att attacken ska göra mer skada? 


\section{Contents}

Acknowledgements $\quad$ ix

List of Abbreviations $\quad$ xi

1 Introduction and Motivation $\quad \mathbf{1}$

1.1 Introduction to Massive MIMO . . . . . . . . . . . . . 1

1.2 Introduction to GNSS . . . . . . . . . . . . . . 2

1.3 Contributions of The Thesis . . . . . . . . . . . . . . 3

1.4 Excluded Papers .................. 5

2 Massive MIMO $\quad 7$

2.1 Training Phase .................... 8

2.2 Uplink Phase . . . . . . . . . . . . . . . . . . 9

2.3 Downlink Phase . . . . . . . . . . . . . . . . 10

2.4 Jamming ..................... 11

3 GNSS 13

3.1 Signal Model . . . . . . . . . . . . . . . . . . . 13

3.2 Position Determination of the GNSS Receiver . . . . . . . . 15

3.3 Spoofing ..................... 16

4 Mathematical Background $\quad 19$

4.1 Detection Theory ................... 19

4.2 Estimation Theory ................... . 21

4.3 Basic Optimization Theory and Power Control Schemes . . . 22

4.3.1 A General Optimization Problem . . . . . . . . . 23

4.3.2 Power Control Schemes ............... 23

5 Conclusions and Future Work $\quad 25$ 
Included Papers

A Is Massive MIMO Robust Against Distributed Jammers? 33

1 Introduction . . . . . . . . . . . . . . . . 35

1.1 Main Contributions . . . . . . . . . . . . . 37

$1.2 \quad$ Notation . . . . . . . . . . . . . . . . . 38

2 System Model . . . . . . . . . . . . . . . . . 38

$2.1 \quad$ Jamming Attack Scenarios . . . . . . . . . . . . . . 39

2.2 Training Phase . . . . . . . . . . . . . . . . 40

3 Channel Estimation . . . . . . . . . . . . . . . 41

4 Uplink Data Transmission . . . . . . . . . . . . . . . . 42

$5 \quad$ MRC Receivers . . . . . . . . . . . . . . . . 44

$5.1 \quad$ First Attack Scenario . . . . . . . . . . . . . . . 44

5.2 Second Attack Scenario . . . . . . . . . . . . . . 45

5.3 Third Attack Scenario . . . . . . . . . . . . . 46

5.4 Fourth Attack Scenario . . . . . . . . . . . . 47

5.5 Comparison of the Attack Scenarios . . . . . . . . 48

$6 \quad$ ZF receiver . . . . . . . . . . . . . . . . 48

7 Optimal Power Control . . . . . . . . . . . . . . . . 49

7.1 Preliminaries for MRC Receiver . . . . . . . . . . . 49

7.2 Optimal Power Control for Jamming-Aware MRC Receiver . . . . . . . . . . . . . . . 49

7.3 Optimal Power Control for Jamming-Ignorant Receiver 52

8 Simulation Results . . . . . . . . . . . . . . . 53

8.1 Evaluation of the Four Attack Scenarios . . . . . . 56

8.2 Massive MIMO versus SIMO with Distributed Jammers 56

8.3 Performance of Massive MIMO with Distributed Jammers 58

8.4 Jamming-Aware Massive MIMO with Power Control . 59

8.5 Jamming-Ignorant Massive MIMO with Power Control 59

8.6 BER Analysis of The First Attack Scenario . . . . . . 62

9 Conclusion . . . . . . . . . . . . . . . 63

10 Appendices ........................ 64

10.1 Appendix A: Proof of Lemma 1 . . . . . . . . . . 64

10.2 Appendix B: Proof of Lemma 2 . . . . . . . . . . . 64

10.3 Appendix C: Proof of Lemma $3 \ldots \ldots 6$

B A Statistical Approach for Multiple Spoofers Detection at 
Mobile GNSS Receivers $\quad 69$

1 Introduction . . . . . . . . . . . . . . 71

1.1 Countermeasure Techniques . . . . . . . . . . 72

1.2 Contributions . . . . . . . . . . . . . . . 73

2 System Model . . . . . . . . . . . . . . . . . . . 74

3 Spoofing Detection . . . . . . . . . . . . . . . 77

3.1 Least-Squares Based Time Delay Estimation for $\mathcal{H}_{1}$ and Signal Acquisition . . . . . . . . . . . . 77

3.2 Least-Squares Based Joint Time Delay and Doppler Frequency Estimation under $\mathcal{H}_{0} \ldots \ldots$. . . . . . . 79

3.3 Complex Amplitudes and AoAs Estimation for the legitimate signals $\left(\mathcal{H}_{0}\right) \ldots \ldots \ldots$. . . . . 80

3.4 Joint Complex Amplitudes and AoAs Estimation and Clustering for the spoofed signals $\left(\mathcal{H}_{1}\right) \ldots \ldots$. . . 81

4 Simulation Results . . . . . . . . . . . . . . . 84

5 Conclusion ................... 87

6 Appendix A: The least-squares based time delay estimation . 87

7 Appendix B: The least-squares based joint time delay and Doppler Frequency estimation . . . . . . . . . . . . . 88

8 Appendix C: Cramer-Rao Bound of the Legitimate Signal . . 89

9 Appendix D: Background on Model Order Selection Rules . . 91

9.1 The Akaike Information Criterion (AIC) Rule . . . . . 91

9.2 The Generalized Information Criterion (GIC) Rule . . 92

9.3 The Bayesian Information Criterion (BIC) Rule . . . . 92 


\section{Acknowledgements}

I would like to send my gratitude to my main supervisor Dr. Erik G. Larsson. For almost two and half years, I have utilized his scientific approaches and discussions. I always enjoy discussing with him. Without the research directions that he has provided to me and his high expectations, I would not be at my current academic level. Likewise, I would like to thank to my co-supervisor Dr. Panos Papadimitratos especially for the fruitful discussions about spoofing and GNSS.

I would also like to thank to Dr. Emil Björnson. I admire and appreciate his kindness, perfection and dedication for research and teaching.

Another special thanks go to Dr. Gökhan Güvensen for his supports and encouragements when I bump into the wall.

To all my colleagues, thank you for the environment that we have established together. I have learned a lot of things from you that will contribute to my rest of life.

Last but not least, I would like to sent great thanks to my family and my aunt for their enormous supports and love.

Ziya Gülgün

Linköping, March 2021 


\section{List of Abbreviations}

$5 \mathrm{G}$

fifth generation of cellular network technology

AWGN additive white Gaussian noise

BIC

Bayesian information criterion

BS

base station

GLRT generalized likelihood ratio test

GPS global positioning system

GNSS global navigation satellite system

i.i.d. independent and identically distributed

LoS line-of-sight

LMMSE linear minimum mean-square error

LS least-squares

MIMO multiple-input multiple-output

ML maximum likelihood

MMSE minimum mean-square error

MRC maximum-ratio-combining

PRN pseudorandom noise

$\mathrm{RF} \quad$ radio frequency

SNR signal-to-noise ratio

SINR signal-to-interference-and-noise ratio

SIMO single-input multiple-output

SE spectral efficiency

TDD time-division duplex

ZF zero forcing 



\section{Chapter 1}

\section{Introduction and Motivation}

Wireless communication has evolved rapidly. Nowadays, not only people communicate with each other, but also people communicate with machines and also machines communicate with each other. Therefore, there exist huge networks and the data rates in these networks have grown exponentially [1]. In order to support these intense demands, communication engineers and researchers should develop innovative but also realistic technologies. While developing new technologies, it is necessary that the vulnerabilities of these technologies are investigated. To motivate this statement, for a moment assume that there is a riot in a city. Some protesters may want to prevent communication among the polices. Therefore, they may want to shut down or disrupt the network by using some low-cost transmitters (jammers, software defined radios, etc.). In this situation, chaos in the city may arise. We anticipate that this type of threat will become more common in the future.

In the next chapters, we introduce two main wireless communication technologies, massive multiple-input multiple-output (MIMO) and global navigation satellite system (GNSS). Moreover, we mention some malicious attacks on these technologies which are mainly jamming attacks for massive MIMO and spoofing attacks for GNSS. We also review some scientific works related to these areas from the literature.

\section{$1.1 \quad$ Introduction to Massive MIMO}

Massive MIMO is a cellular network technology that was proposed for $5 \mathrm{G}$ [2], [3] and is now commercially deployed [4]. Basically, in this technology, a base station (BS) having a massive number of antenna elements, around one hundred, supported by independent radio frequency (RF) chains serves many 
terminals simultaneously in the same time-frequency resource. Massive MIMO can serve the terminals concurrently because there exist two fundamental phenomenon that are the result of the law of large numbers [5]: channel hardening and favorable propagation [6]. These two phenomenon will be introduced later.

Since massive MIMO is one of the main physical layer technologies used in $5 \mathrm{G}$, it is required to analyze the vulnerabilities of this technology to malicious attacks, such as eavesdropping and jamming, and to compare with those of previous generations cellular systems. For example in [7], it is shown that the spectral efficiency (SE) between the BS and passive eavesdropper remains same when the number of antennas at the BS increases, though the $\mathrm{SE}$ between the BS and the legitimate user increases. Therefore, in [7] it is concluded that massive MIMO is robust to passive eavesdropping, since only the legitimate communication benefits from the array gain. However, it is also shown that an active attack, like jamming, on the BS during the training phase can be harmful [7].

Jammers are able to send any signals. For example, in [8], the jammers are assumed to know the pilot signals and the vulnerabilities of massive MIMO are analyzed when the jammers send the pilot signals to the BS during training phase. In [9-11], some countermeasure techniques are proposed when the jammers send the pilot signals as the attack signals. Jammers may also send random attack signals. In [12], a jamming-resistant massive MIMO is proposed when the jammer transmits random signals. In $[13,14]$, the jammers transmit random attack signals.

When we look at the literature, some unanswered questions exist: Is massive MIMO more vulnerable or more resistant to jamming attacks than current cellular systems? Do we need to spread the jamming power over the space? If there exist multiple jammers in the environment, what is the best attack strategy for the jammers? In Paper A, we answer these questions.

\subsection{Introduction to GNSS}

A wireless technology that provides position, time and velocity to users is GNSS. [15]. Some countries have their own GNSS. For example, the United States uses the global positioning system (GPS). The European Union uses the Galileo navigation system. GNSS is the general name of the navigation systems and it includes both the GPS and Galileo.

For the last decade, the usage of GNSS technology has tremendously increased. Especially today's evolving technologies, such as unmanned aerial 
vehicles and cars, are strongly relying on GNSS to obtain position information. Therefore, providing secure GNSS technology is extremely crucial. However, GNSS technology is vulnerable to malicious attacks, more specifically spoofing in which the malicious device identifies itself as a legitimate device. In $[16,17]$, the vulnerabilities of GNSS to the spoofing are evaluated. With today's lowcost software defined radio technology, one can easily spoof GNSS receivers [18]. Therefore, effective countermeasure techniques should be presented for spoofing attacks.

In the literature, there exist several countermeasure techniques for spoofing attacks and we can classify these techniques as follows:

Signal Processing Techniques: In [19,20], detectors are derived that utilize the fact that GNSS receivers obtain the same position information when they are affected by the same spoofer. In [21], a new statistical test is proposed for the presence of multiple spoofers based on range measurements observed by multiple GNSS receivers located on a rigid body platform. In [22,23], detectors are proposed based on the correlation among the received signals. In [24], a countermeasure technique for spoofing is developed based on subspace projection.

Encryption Techniques: In [25,26], novel navigation message authentication schemes for the GNSS signals are given. The technique proposed in [25] combines two authentications of GPS, namely cryptographic authentication and signal timing authentication based on statistical hypothesis tests. In [27], a technique is presented for detecting spoofing attacks against cryptographically-secured GNSS.

In Paper B, we propose a detection method for the mobile GNSS receiver to detect the multiple spoofers. This method falls into the signal processing category. The strong and novel aspect of this work is that the detector can operate if the GNSS receiver does not know the number of spoofers in the environment and even if the GNSS receiver does not know the attack strategy of the spoofers. A limitation is that at least two legitimate signals should be spoofed by the same spoofer.

\subsection{Contributions of The Thesis}

The thesis has two main contributions. Firstly, we analyze the effects of the distributed jammers on massive MIMO in Paper A. Secondly, we propose a countermeasure technique for the mobile GNSS receivers, that detect multiple spoofers attack and this technique is presented in Paper B. 


\section{Paper A: Is Massive MIMO Robust Against Distributed Jammers?}

Authored by: Ziya Gülgün, Emil Björnson, and Erik G. Larsson

Published in: IEEE Transactions on Communications, vol. 69, no. 1, pp. 457-469, January 2021.

Abstract: In this paper, we evaluate the uplink spectral efficiency (SE) of a single-cell massive multiple-input-multiple output (MIMO) system with distributed jammers. We define four different attack scenarios and compare their impact on the massive MIMO system as well as on a conventional single-input multiple-output (SIMO) system. More specifically, the jammers attack the base station (BS) during both the uplink training phase and data phase. The BS uses either least squares (LS) or linear minimum mean square error (LMMSE) estimators for channel estimation and utilizes either maximum-ratio-combining (MRC) or zero-forcing (ZF) decoding vectors. We show that ZF gives higher SE than MRC but, interestingly, the performance is unaffected by the choice of the estimators. The simulation results show that the performance loss percentage of massive MIMO is less than that of the SIMO system. Moreover, we consider two types of power control algorithms: jamming-aware and jamming-ignorant. In both cases, we consider the maxmin and proportional fairness criteria to increase the uplink SE of massive MIMO systems. We notice numerically that max-min fairness is not a good option because if one user is strongly affected by the jamming, it will degrade the other users' SE as well. On the other hand, proportional fairness improves the sum SE of the system compared with the full power transmission scenario.

\section{Paper B: A Statistical Approach for Multiple Spoofers Detection at Mobile GNSS Receivers}

Authored by: Ziya Gülgün, Erik G. Larsson, Panos Papadimitratos

Submitted to IEEE Transactions on Aerospace and Electronic Systems.

Abstract: We consider global navigation satellite system (GNSS) spoofing attacks and devise a countermeasure appropriate for mobile GNSS receivers. Our approach is to design detectors that, operating after the signal acquisition, enable the victim receiver to determine with high probability whether it is under a spoofing attack or not. Namely, the binary hypothesis is that either the GNSS receiver tracks legitimate satellite signals, $\mathcal{H}_{0}$, or spoofed signals, $\mathcal{H}_{1}$. We assume that there exists an unknown number of multiple spoofers in the environment and the attack strategy (which legitimate signals are spoofed by which spoofers) is not known to the receiver. Based on these 
assumptions, we propose an algorithm that identifies the number of spoofers and clusters the spoofing data by using Bayesian information criteria (BIC) rules. Depending on the estimated and clustered data we propose a detector, called as generalized likelihood ratio (GLRT)-like detector. We compare the performance of the GLRT-like detector with a genie-aided detector in which the attack strategy and the number of spoofers is known by the receiver.

\subsection{Excluded Papers}

The papers in Table 1 are excluded from the thesis because they are the conference versions of Paper A and Paper B, respectively. 
Z. Gülgün, E. Björnson, and E. G. Larsson, "Performance Analysis of Massive MIMO With Distributed Jammers," in 2020 IEEE International Conference on Communications (ICC), Dublin, Ireland, 2020, pp. 1-6.

Z. Gülgün, E. G. Larsson, and P. Papadimitratos, "Statistical method for spoofing detection at mobile GNSS receivers," in 2019 16th International Symposium on Wireless Communication Systems (ISWCS), Oulu, Finland, 2019, pp. 677-681. 


\section{Chapter 2}

\section{Massive MIMO}

A single-cell massive MIMO system consists of a BS having $M$ antennas and $K$ single-antenna users. We assume that each antenna in the BS has one RF chain. Therefore, the BS can digitally control the relative phase between all of its antenna elements.

A wireless channel is a medium in which the data can be transmitted and received. The characteristics of wireless channels may vary depending on the time and frequency. We can describe these changes in terms of two main concepts [28]: Large scale fading, due to path loss of signal as a function of distance, frequency and shadowing by large obstacles, and small scale fading, due to the constructive and destructive interference of the multiple signal paths between the transmitter and receiver. The coherence block is a block where small scale fading can be assumed to be constant and the channel model is called as block-fading model.

The channel between the BS and the $k^{\text {th }}$ user including multipath propagation, attenuation, and shadowing, can be characterized as a complex vector, $\mathbf{g}_{k} \in \mathbb{C}^{M}$, based on the baseband representation of the signals $[29,30]$. Depending on the channel models, such as Rayleigh, Rician fading and line-ofsight (LoS) models, $\mathbf{g}_{k}$ can be characterized $[6,28,31]$. Throughout this thesis, we only utilize the Rayleigh fading channel model, i.e., $\mathbf{g}_{k} \sim \mathcal{C N}\left(\mathbf{0}, \beta_{k} \mathbf{I}\right)$ where $\beta_{k}$ is the large scale fading coefficient corresponding to the $k^{t h}$ user. Moreover, the channel statistic for each user is independent of each other.

The channel vector has dimension of $M$. By using the law of large numbers, we can obtain the following results:

$$
\frac{\left\|\mathbf{g}_{k}\right\|^{2}}{\mathbb{E}\left\{\left\|\mathbf{g}_{k}\right\|^{2}\right\}} \stackrel{\text { a.s. }}{\rightarrow} 1, \quad \text { as } M \rightarrow \infty
$$




$$
\frac{\mathbf{g}_{k}^{\mathrm{H}} \mathbf{g}_{k^{\prime}}}{\sqrt{\mathbb{E}\left\{\left\|\mathbf{g}_{k}\right\|^{2}\right\} \mathbb{E}\left\{\left\|\mathbf{g}_{k^{\prime}}\right\|^{2}\right\}}} \stackrel{\text { a.s. }}{\rightarrow} 0, \quad \text { as } M \rightarrow \infty, \quad k \neq k^{\prime} .
$$

The result in (1) is called channel hardening [6,32]. It means that in the limit case of $M$, the channel behaves deterministically. The result in (2) is called favorable propagation which implies that in the limit case of $M$, the channels of two different users are orthogonal to each other $[6,32]$. These are two fundamental phenomena in massive MIMO. Obviously, the asymptotic behavior in $M$ has no physical meaning. However, these results give some intuitions for massive MIMO where $M$ is large.

In this thesis, the block Rayleigh fading model is used. In the next sections, we explain how this block is divided for the data transmissions: training, uplink and downlink phases.

\subsection{Training Phase}

Let us assume that the length of coherence block is $T$. Some sub-block, say $\tau$, from the coherence block should be reserved for channel estimation. In the training phase, the users send orthonormal vectors to the BS such that:

$$
\left\|\phi_{k}\right\|^{2}=1, \quad \phi_{k}^{\mathrm{H}} \phi_{k^{\prime}}=0 \quad k \neq k^{\prime} .
$$

Therefore, the received signal in the BS can be expressed as:

$$
\mathbf{Y}=\sum_{k=1}^{K} \sqrt{\tau \rho_{u l}} \mathbf{g}_{k} \boldsymbol{\phi}_{k}^{T}+\mathbf{N},
$$

where $\mathbf{Y}$ and $\mathbf{N}$ are $M \times \tau$ received signal and noise matrices, respectively. The elements of $\mathbf{N}$ are independent and identically distributed (i.i.d.) random variables characterized by $\mathcal{C N}\left(0, \sigma^{2}\right)$. $\rho_{u l}$ is the transmit power.

To estimate the $k^{t h}$ user's channel, the BS processes the received signal as:

$$
\mathbf{y}_{k}=\mathbf{Y} \boldsymbol{\phi}_{k}^{*}=\sqrt{\tau \rho_{u l}} \mathbf{g}_{k}+\mathbf{n},
$$

where $\mathbf{n}$ is a noise vector whose entries are i.i.d. again with $\mathcal{C N}\left(0, \sigma^{2}\right)$ because the unitary multiplication does not change the statistics of the Gaussian distribution.

By assumption, the large scale fading coefficient, $\beta_{k}$, is known. Therefore, the minimum mean-square error (MMSE) estimator of $\mathbf{g}_{k}$ can be written [33]:

$$
\mathbf{g}_{k}^{\mathrm{MMSE}}=\frac{\sqrt{\tau \rho_{u l}} \beta_{k}}{\sigma^{2}+\tau \rho_{u l} \beta_{k}} \mathbf{y}_{k} .
$$


Another estimator is the least-squares (LS). The LS estimator can be expressed as [33]:

$$
\mathbf{g}_{k}^{\mathrm{LS}}=\frac{\mathbf{y}_{k}}{\sqrt{\tau \rho_{u l}}} .
$$

When we compare the (6) and (7), the only difference is the scaling factor. These estimators are used for decoder and precoder designs by the BS.

\subsection{Uplink Phase}

In the uplink phase, the users send the data to the BS. The $k^{\text {th }}$ terminal transmits the following weighted symbol:

$$
x_{k}=\sqrt{\eta_{k}} q_{k},
$$

where $q_{k}$ is the generated symbol with zero mean and unit variance and $\eta_{k}$ is the power control coefficient satisfying $0 \leq \eta_{k} \leq 1$. Moreover, the symbols transmitted by all users are uncorrelated. Based on this model, the received signal vector can be expressed as:

$$
\mathbf{y}=\sqrt{\rho_{u l}} \sum_{k=1}^{K} \sqrt{\eta_{k}} \mathbf{g}_{k} x_{k}+\mathbf{n} .
$$

The BS creates normalized decoder vectors, $\mathbf{a}_{k}$, based on the imperfect channel estimates to infer each user's transmitted data. After decoding, the signal corresponding to the $k^{\text {th }}$ user can be written as:

$$
\begin{aligned}
r_{k} & =\mathbf{a}_{k}^{\mathrm{H}} \mathbf{y} \\
& =\sqrt{\rho_{u l} \eta_{k}} \mathbb{E}\left\{\mathbf{a}_{k}^{\mathrm{H}} \mathbf{g}_{k}\right\} x_{k}+\sqrt{\rho_{u l} \eta_{k}}\left(\mathbf{a}_{k}^{\mathrm{H}} \mathbf{g}_{k}-\mathbb{E}\left\{\mathbf{a}_{k}^{\mathrm{H}} \mathbf{g}_{k}\right\}\right) x_{k} \\
& +\sum_{k^{\prime}=1, k^{\prime} \neq k}^{K} \sqrt{\rho_{u l} \eta_{k^{\prime}}} \mathbf{a}_{k}^{\mathrm{H}} \mathbf{g}_{k^{\prime}} x_{k^{\prime}}+\mathbf{a}_{k}^{\mathrm{H}} \mathbf{n} .
\end{aligned}
$$

When we observe the four terms in (10), they are uncorrelated. Therefore, we can consider the first term as the desired signal and the remaining terms as effective noise. After this, the effective signal-to-interference-and-noise ratio (SINR) can be written:

$$
\operatorname{SINR}_{k}^{\mathrm{UL}}=\frac{\rho_{u l} \eta_{k}\left|\mathbb{E}\left\{\mathbf{a}_{k}^{\mathrm{H}} \mathbf{g}_{k}\right\}\right|^{2}}{\sum_{k^{\prime}=1}^{K} \rho_{u l} \eta_{k^{\prime}} \mathbb{E}\left\{\left|\mathbf{a}_{k}^{\mathrm{H}} \mathbf{g}_{k^{\prime}}\right|^{2}\right\}-\rho_{u l} \eta_{k}\left|\mathbb{E}\left\{\mathbf{a}_{k}^{\mathrm{H}} \mathbf{g}_{k}\right\}\right|^{2}+\sigma^{2} \mathbb{E}\left\{\left\|\mathbf{a}_{k}\right\|^{2}\right\}}
$$


If we apply capacity-bounding techniques, we can obtain the following:

$$
C_{k}^{\mathrm{UL}} \geq \log _{2}\left(1+\mathrm{SINR}_{k}^{\mathrm{UL}}\right)
$$

where $C_{k}^{\mathrm{UL}}$ is the uplink capacity. The bounding technique is called use-andthen-forget bound in [6]. The theory behind use-and-then-forget bound is the Jensen's inequality [34] and the fact that the channel estimation information is not used as side information to calculate SE.

There are two main decoder vectors that are widely used in the massive MIMO literature [6,31]: maximum-ratio-combining (MRC) and zero forcing $(\mathrm{ZF})$. These decoder vectors are explicitly written as:

$$
\begin{gathered}
\left(\mathbf{a}_{k}^{\mathrm{MRC}}\right)^{\mathrm{H}}=\hat{\mathbf{g}}_{k}^{\mathrm{H},} \\
\left(\mathbf{a}_{k}^{\mathrm{ZF}}\right)^{\mathrm{H}}=\left(\hat{\mathbf{G}}^{\mathrm{H}} \hat{\mathbf{G}}\right)_{k^{t h} \text { row }}^{-1} \hat{\mathbf{G}}^{\mathrm{H}},
\end{gathered}
$$

where $\hat{\mathbf{g}}_{k}$ is any channel estimate vector either in (6) or (7), $\hat{\mathbf{G}}$ is an $M \times K$ matrix whose $k^{t h}$ column is $\hat{\mathbf{g}}_{k}$.

\subsection{Downlink Phase}

In the downlink phase, the BS generates symbols for each user, $q_{k}$, which are zero mean, unit variance and uncorrelated with each other. To transmit these symbols, the BS creates the normalized precoder vectors such that:

$$
\mathbf{x}=\sqrt{\rho_{d l}} \sum_{k=1}^{K} \sqrt{\eta_{k}} \mathbf{p}_{k} q_{k}
$$

where $\mathbf{x}$ and $\mathbf{p}_{k}$ are the $M \times 1$ transmitted signal and the precoder vector, $\left\|\mathbf{p}_{k}\right\|^{2}=1$, respectively, and $\eta_{k}$ is the power control coefficient satisfying:

$$
\sum_{k=1}^{K} \eta_{k}=1
$$

For the precoding vectors, the BS can utilize the channel estimates because massive MIMO operates in time-division duplex (TDD) mode, which implies that the uplink and downlink channels are reciprocal. The precoder vectors are generally chosen as maximum-ratio or ZF precoders.

The $k^{t h}$ user receives the following signal:

$$
z_{k}=\sqrt{\rho_{d l} \eta_{k}} \mathbf{g}_{k}^{T} \mathbf{p}_{k} q_{k}+\sqrt{\rho_{d l}} \sum_{k^{\prime}=1, k^{\prime} \neq k}^{K} \sqrt{\eta_{k^{\prime}}} \mathbf{g}_{k^{\prime}}^{T} \mathbf{p}_{k^{\prime}} q_{k^{\prime}}+n
$$


where $n$ is the noise with $\mathcal{C N}\left(0, \sigma^{2}\right)$. Applying the same manipulation as in (10), we obtain:

$$
\begin{aligned}
z_{k}= & \sqrt{\rho_{d l} \eta_{k}} \mathbb{E}\left\{\mathbf{g}_{k}^{T} \mathbf{p}_{k}\right\} q_{k}+\sqrt{\rho_{d l} \eta_{k}}\left(\mathbf{g}_{k}^{T} \mathbf{p}_{k}-\mathbb{E}\left\{\mathbf{g}_{k}^{T} \mathbf{p}_{k}\right\}\right) q_{k} \\
& \sqrt{\rho_{d l}} \sum_{k^{\prime}=1, k^{\prime} \neq k}^{K} \sqrt{\eta_{k^{\prime}}} \mathbf{g}_{k^{\prime}}^{T} \mathbf{p}_{k^{\prime}} q_{k^{\prime}}+n,
\end{aligned}
$$

and the effective SINR is:

$$
\operatorname{SINR}_{k}^{\mathrm{DL}}=\frac{\rho_{d l} \eta_{k}\left|\mathbb{E}\left\{\mathbf{g}_{k}^{T} \mathbf{p}_{k}\right\}\right|^{2}}{\sum_{k^{\prime}=1}^{K} \rho_{d l} \eta_{k^{\prime}} \mathbb{E}\left\{\left|\mathbf{g}_{k}^{T} \mathbf{p}_{k^{\prime}}\right|^{2}\right\}-\rho_{d l} \eta_{k}\left|\mathbb{E}\left\{\mathbf{g}_{k}^{T} \mathbf{p}_{k}\right\}\right|^{2}+\sigma^{2}} .
$$

If we apply the use-and-then-forget bound technique, we obtain:

$$
C_{k}^{\mathrm{DL}} \geq \log _{2}\left(1+\mathrm{SINR}_{k}^{\mathrm{DL}}\right)
$$

where $C_{k}^{\mathrm{DL}}$ is the downlink capacity.

\subsection{Jamming}

In this chapter, we analyze the effect of jamming on massive MIMO. Suppose there is a jammer in the single-cell massive MIMO environment, that attacks the BS in the training and the uplink phase. The jammer may also attack any users but the aim of jamming is to collapse the whole network. The received signal in (4) can be rewritten as:

$$
\mathbf{Y}=\sum_{k=1}^{K} \sqrt{\tau \rho_{u l}} \mathbf{g}_{k} \boldsymbol{\phi}_{k}^{T}+\rho_{j} \mathbf{h}_{j} \mathbf{j}^{T}+\mathbf{N}
$$

where $\mathbf{h}_{j}$ is the $M \times 1$ channel vector for the jammer, modeled as Rayleigh fading with variance $\beta_{j}, \rho_{j}$ is the power of jamming signal, and $\mathbf{j}$ is the jamming vector of dimension $\tau \times 1$. In general, the jamming signal is modeled by Gaussian signals so $\mathbf{j}$ is characterized as $\mathcal{C N}(\mathbf{0}, \mathbf{I})$. The projected signal can be re-expressed:

$$
\mathbf{y}_{k}=\mathbf{Y} \boldsymbol{\phi}_{k}^{*}=\sqrt{\tau \rho_{u l}} \mathbf{g}_{k}+\sqrt{\rho_{j}} \mathbf{h}_{j} \mathbf{j}^{T} \boldsymbol{\phi}_{k}^{*}+\mathbf{n} .
$$

From (22), it is clear that the projected signal is contaminated by the jamming signals and it affects the channel estimation quality of $\mathbf{g}_{k}$. 
A closed form MMSE estimator of $\mathbf{g}_{k}$ does not exist in general because the noise term including the jamming part in (22) is not Gaussian. Therefore, we present the linear minimum mean-square error (LMMSE) estimator of $\mathbf{g}_{k}$ as [33]:

$$
\mathbf{g}_{k}^{\mathrm{LMMSE}}=\frac{\sqrt{\tau \rho_{u l}} \beta_{k}}{\sigma^{2}+\tau \rho_{u l} \beta_{k}+\rho_{j} \beta_{j}} \mathbf{y}_{k},
$$

It is important to note that the BS should know or estimate the power of the received jamming signal which appears in the scaling factor in (23).

The LS estimator is:

$$
\mathbf{g}_{k}^{\mathrm{LS}}=\frac{\mathbf{y}_{k}}{\sqrt{\tau \rho_{u l}}} .
$$

Again, the fundamental difference between these two estimators is a scaling factor.

Now let us focus on the uplink phase. The received signal under the jammer attack can be presented:

$$
\mathbf{y}=\sqrt{\rho_{u l}} \sum_{k=1}^{K} \sqrt{\eta_{k}} \mathbf{g}_{k} x_{k}+\sqrt{\rho_{j}} \mathbf{h}_{j} s+\mathbf{n},
$$

where $s$ is the attack signal with distribution $\mathcal{C N}(0,1)$. Based on the signal model in (25), the effective SINR is written:

$\mathrm{SINR}_{k}^{\mathrm{UL}, j}=$

$$
\frac{\rho_{u l} \eta_{k}\left|\mathbb{E}\left\{\mathbf{a}_{k}^{\mathrm{H}} \mathbf{g}_{k}\right\}\right|^{2}}{\sum_{k^{\prime}=1}^{K} \rho_{u l} \eta_{k^{\prime}} \mathbb{E}\left\{\left|\mathbf{a}_{k}^{\mathrm{H}} \mathbf{g}_{k^{\prime}}\right|^{2}\right\}-\rho_{u l} \eta_{k}\left|\mathbb{E}\left\{\mathbf{a}_{k}^{\mathrm{H}} \mathbf{g}_{k}\right\}\right|^{2}+\rho_{j} \mathbb{E}\left\{\left|\mathbf{a}_{k}^{\mathrm{H}} \mathbf{h}_{j}\right|^{2}\right\}+\sigma^{2} \mathbb{E}\left\{\left\|\mathbf{a}_{k}\right\|^{2}\right\}}
$$

From (26), we observe that there is additional interference in the denominator because of the jamming signal. Moreover, the decoder vector, $\mathbf{a}_{k}$, is contaminated by the jammer. Therefore,

$$
\operatorname{SINR}_{k}^{\mathrm{UL}}>\mathrm{SINR}_{k}^{\mathrm{UL}, j} .
$$

In Paper A, we analyze the effects of the distributed jammers on massive MIMO for different power control schemes. 


\section{Chapter 3}

\section{GNSS}

\subsection{Signal Model}

GNSS describes the collection of satellite positioning systems. The most famous satellite positioning system is GPS belonging to the United States. GLONASS, another satellite system, is operated by Russia. Galileo is a civil GNSS system operated by the European Global Navigation Satellite Systems Agency. China, Japan and India have GNSS systems as well. The working principles of satellite positioning systems are almost same [15]. Therefore, we present a general GNSS system structure.

Suppose there are $M$ visible satellites in the environment. A typical complex baseband received GNSS signal can be expressed:

$$
y(t)=\sum_{m=1}^{M} A_{m} D_{m}\left(t-\tau_{m}\right) C_{m}\left(t-\tau_{m}\right) \exp \left(j 2 \pi\left(f_{m}^{d} t-f_{c} \tau_{m}\right)\right)
$$

where $A_{m}$ is the complex amplitude, $D_{m}(t)$ is the message signal, $\tau_{m}$ is the propagation delay, $C_{m}(t)$ is the spreading code, generally chosen from pseudorandom noise (PRN) codes, $f_{m}^{d}$ is the Doppler frequency corresponding to the $m^{t h}$ satellite and $f_{c}$ is the carrier frequency. It is important to note that the propagation delay appears as a phase shift in (28).

PRN codes are quasi-orthogonal with each other. Mathematically, we can express this property as:

$$
R\left(\tau_{m}-\tau_{k}\right) \delta_{m k} \approx \int C_{m}\left(t-\tau_{m}\right) C_{k}^{*}\left(t-\tau_{k}\right) d t
$$

where $\delta_{m k}$ is the Kronecker delta function, and $R(\tau)$ is the auto-correlation function of any PRN codes. If we normalize the PRN codes, $R(\tau)=1$ for 


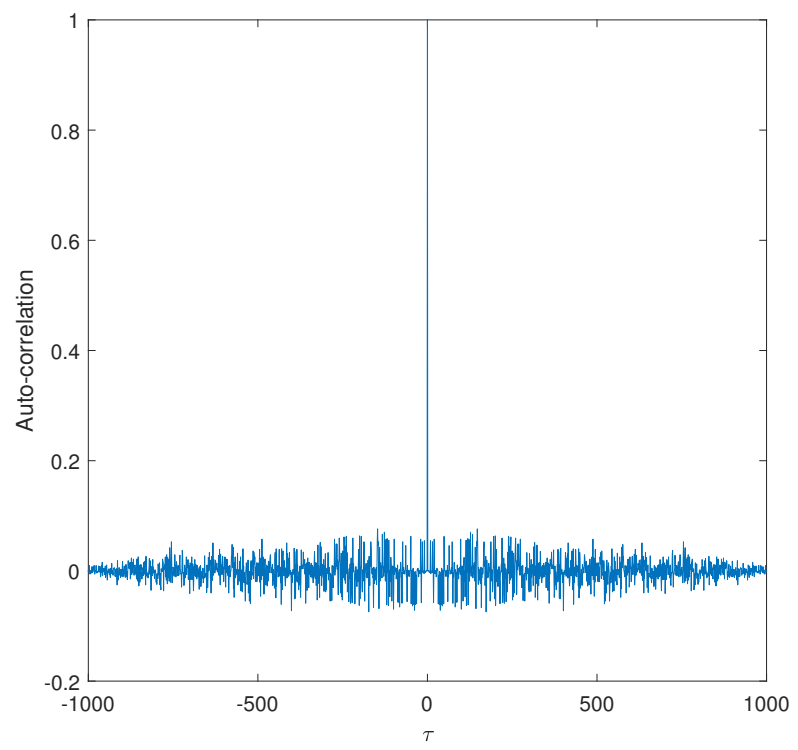

Figure 1: Absolute value of the auto-correlation function of a PRN code.

$\tau=0$. To visualize the quasi-orthogonality property, we present the absolute value of auto-correlation and cross-correlation functions of PRN codes in Figure 1 and Figure 2, respectively. As illustrated in Figure 2, the crosscorrelation function is very close to zero, but never becomes zero. However, when we compare the values of cross-correlation function and the peak value of the auto-correlation function, we can conclude that the PRN codes are quasi-orthogonal.

The propagation delay, $\tau$, is used to calculate the geometric range between the user and the satellite. Therefore, the GNSS receiver should estimate the propagation delay. By using the quasi-orthogonality property, the nonlinear least-squares (LS) estimate of the propagation delay and the Doppler frequency can be written as:

$$
\hat{f}_{m}^{d}, \hat{\tau}_{m} \approx \underset{f, \tau}{\operatorname{argmax}}\left|\int y^{*}(t) C_{m}(t-\tau) e^{j 2 \pi f t} d t\right| .
$$

In the next section, we explain the position calculation of the GNSS receiver. 


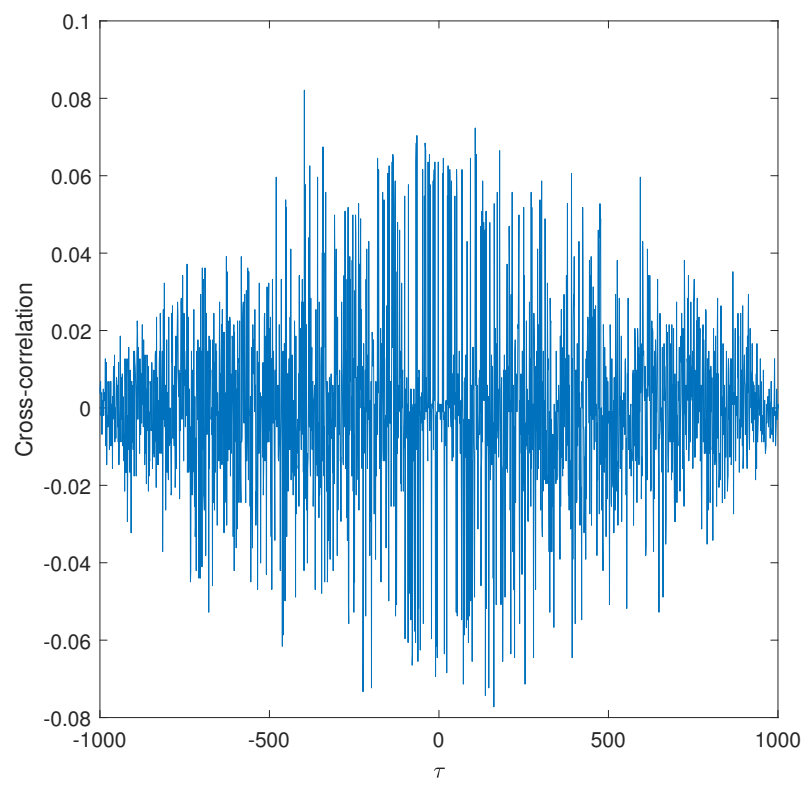

Figure 2: Absolute value of the cross-correlation function of two PRN codes.

\subsection{Position Determination of the GNSS Receiver}

We assume that the propagation delay is perfectly estimated. The geometric range between the $m^{\text {th }}$ satellite and the user can be expressed as:

$$
r_{m}=c \tau_{m},
$$

where $c$ is the speed of light. However, the measurement in the GNSS receiver is different from $r_{m}$ because of the clock offset in the satellite and in the GNSS receiver. This measurement is called pseudorange and can be expressed as [15]:

$$
\rho_{m}=r_{m}+c\left(t_{u}-\delta t_{m}\right),
$$

where $t_{u}$ and $\delta t_{m}$ are the GNSS receiver and the satellite clock offsets, respectively. The satellite clock offset, $\delta t_{m}$, is the result of bias and drift contributions. A system ground monitoring network (SATNAV) determines corrections for these offsets and transmits corrections to the satellites. The satellites rebroadcast these corrections to the users in the navigation message. Therefore, the pseudorange can be expressed as:

$$
\rho_{m}=r_{m}+c t_{u} .
$$


For a 3-dimensional environment, $r_{m}$ can explicitly be written as:

$$
r_{m}=\sqrt{\left(x_{m}-x_{u}\right)^{2}+\left(y_{m}-y_{u}\right)^{2}+\left(z_{m}-z_{u}\right)^{2}},
$$

where $\left\{x_{m}, y_{m}, z_{m}\right\}$ and $\left\{x_{u}, y_{u}, z_{u}\right\}$ are the coordinates of the $m^{\text {th }}$ satellite and the GNSS receiver based on the center of earth, respectively. In general, four reference satellites are sufficient for position calculation [15]. We can obtain the following set of non-linear equations:

$$
\begin{aligned}
& \rho_{1}=\sqrt{\left(x_{1}-x_{u}\right)^{2}+\left(y_{1}-y_{u}\right)^{2}+\left(z_{1}-z_{u}\right)^{2}}+c t_{u}+\epsilon_{1}, \\
& \rho_{2}=\sqrt{\left(x_{2}-x_{u}\right)^{2}+\left(y_{2}-y_{u}\right)^{2}+\left(z_{2}-z_{u}\right)^{2}}+c t_{u}+\epsilon_{2}, \\
& \rho_{3}=\sqrt{\left(x_{3}-x_{u}\right)^{2}+\left(y_{3}-y_{u}\right)^{2}+\left(z_{3}-z_{u}\right)^{2}}+c t_{u}+\epsilon_{3}, \\
& \rho_{4}=\sqrt{\left(x_{4}-x_{u}\right)^{2}+\left(y_{4}-y_{u}\right)^{2}+\left(z_{4}-z_{u}\right)^{2}}+c t_{u}+\epsilon_{4},
\end{aligned}
$$

where $\epsilon_{i}$ is the measurement noise in $\rho_{i}$. These non-linear equations can be solved based on linearization techniques and iterative methods such as the Kalman filter and extended Kalman filter [35]. These iterative techniques are out of the scope of this work.

After calculation of the user position, it is easy to find the average velocity:

$$
v=\frac{\left\|\mathbf{u}\left(t_{2}\right)-\mathbf{u}\left(t_{1}\right)\right\|}{t_{2}-t_{1}}
$$

where $\mathbf{u}(t)$ is the vector that contains the user position at time $t$.

\subsection{Spoofing}

Spoofing of GNSS is the broadcast of fake signals with the intent that the victim receiver identifies them as authentic signals. Since the standards of GNSS systems, for example GPS, are publicly available and low-cost radio systems can be accessible, it is easy to spoof a GNSS receiver.

A spoofer transmits falsified signals such as:

$$
y^{s}(t)=\sum_{m=1}^{N} A_{m}^{s} D_{m}^{s}\left(t-\tau_{m}^{s}\right) C_{m}\left(t-\tau_{m}^{s}\right) \exp \left(j 2 \pi\left(f_{m}^{d, s} t-f_{c} \tau_{m}^{s}\right)\right)
$$

where $N$ is the number of spoofed signals, $A_{m}^{s}, D_{m}^{s}, \tau_{m}^{s}, f_{m}^{d, s}$ are the complex amplitude, the falsified message signal, the propagation delay and the Doppler 
frequency of the spoofed signal, respectively. From (40), the PRN codes that are used by the satellites in (28) appear in the spoofed signals. It is important to note that the falsified signals, $D_{m}^{s}$, give fake position, time and velocity information to the GNSS receiver.

In Paper B, we propose a detector for the mobile GNSS receiver, which does not have any prior knowledge about the number of spoofers or which legitimate signals are spoofed by which spoofer. 


\section{Chapter 4}

\section{Mathematical Background}

\subsection{Detection Theory}

We consider a simple hypothesis testing problem which means we know that either $\mathcal{H}_{0}$ or $\mathcal{H}_{1}$ is true. There are four possible combinations of events that can occur:

- $\mathcal{H}_{0}$ true; choose $\mathcal{H}_{0}$

- $\mathcal{H}_{0}$ true; choose $\mathcal{H}_{1}$

- $\mathcal{H}_{1}$ true; choose $\mathcal{H}_{1}$

- $\mathcal{H}_{1}$ true; choose $\mathcal{H}_{0}$

The important question is how do we establish a decision rule? One decision criterion is the Bayes criterion that minimizes risk function [36]:

$$
\begin{aligned}
& R=C_{00} P_{0} \operatorname{Pr}\left(\text { decide } \quad \mathcal{H}_{0} \mid \mathcal{H}_{0} \quad \text { is true }\right)+ \\
& C_{10} P_{0} \operatorname{Pr}\left(\text { decide } \quad \mathcal{H}_{1} \mid \mathcal{H}_{0} \text { is true }\right)+ \\
& C_{11} P_{1} \operatorname{Pr}\left(\text { decide } \quad \mathcal{H}_{1} \mid \mathcal{H}_{1} \text { is true }\right)+ \\
& C_{01} P_{1} \operatorname{Pr}\left(\text { decide } \mathcal{H}_{0} \mid \mathcal{H}_{1}\right. \text { is true), }
\end{aligned}
$$

where $C_{00}, C_{10}, C_{11}$ and $C_{01}$ are the positive-valued cost functions corresponding to four different observations, $P_{0}$ and $P_{1}$ are prior probabilities for $\mathcal{H}_{0}$ and $\mathcal{H}_{1}$, respectively, and $\operatorname{Pr}(\mid)$ denotes the conditional probability.

Now we have an $N$ dimension of observation vector, $\mathbf{r}$, with the following conditional probability density functions: $p\left(\mathbf{r} \mid \mathcal{H}_{0}\right)$ and $p\left(\mathbf{r} \mid \mathcal{H}_{1}\right)$. The decision 
rule that minimizes the risk function in (41) can be expressed as [36]:

$$
\begin{gathered}
\mathcal{H}_{1} \\
\frac{p\left(\mathbf{r} \mid \mathcal{H}_{1}\right)}{\overline{p\left(\mathbf{r} \mid \mathcal{H}_{0}\right)}}>^{>}{ }^{<} \\
\mathcal{H}_{0}
\end{gathered}
$$

where $\eta$ is defined as:

$$
\eta=\frac{P_{0}\left(C_{10}-C_{00}\right)}{P_{1}\left(C_{01}-C_{11}\right)}
$$

Based on (42), it is clear that the Bayes decision criterion leads to a likelihood ratio test.

In the literature, the likelihood ratio test is commonly implemented by taking the natural logarithm:

$$
\log \left(\frac{p\left(\mathbf{r} \mid \mathcal{H}_{1}\right)}{p\left(\mathbf{r} \mid \mathcal{H}_{0}\right)}\right) \underset{\substack{\mathcal{H}_{1} \\ \mathcal{H}_{0}}}{>} \log (\eta) .
$$

Since the natural logarithm is monotonic function and both sides of (42) are positive, the decision criteria in (42) and (44) are equivalent to each other.

Someone may not be so lucky that they obtain prior probabilities of the hypotheses. Let us assume that a target exists in $\mathcal{H}_{1}$ and the target is not present in $\mathcal{H}_{0}$. In such a case, one approach is to maximize the probability of detection, $\operatorname{Pr}\left(\right.$ decide $\quad \mathcal{H}_{1} \mid \mathcal{H}_{1}$ is true $)=P_{D}$, or minimize the probability of miss, $\operatorname{Pr}\left(\right.$ decide $\quad \mathcal{H}_{0} \mid \mathcal{H}_{1}$ is true $)=P_{M}$, with a constant false alarm rate, $\operatorname{Pr}\left(\right.$ decide $\quad \mathcal{H}_{1} \mid \mathcal{H}_{0}$ is true $)=P_{F}=\alpha$. By using the Lagrange multipliers, we can maximize the following function [37]:

$$
F=P_{D}-\lambda\left(P_{F}-\alpha\right) .
$$

$P_{D}$ and $P_{F}$ can explicitly written:

$$
\begin{aligned}
& P_{D}=\int_{Z_{1}} p\left(\mathbf{r} \mid \mathcal{H}_{1}\right) d \mathbf{r} \\
& P_{F}=\int_{Z_{1}} p\left(\mathbf{r} \mid \mathcal{H}_{0}\right) d \mathbf{r}
\end{aligned}
$$

where $Z_{1}$ is the decision region for $\mathcal{H}_{1}$. Equation (45) can be rewritten:

$$
F=\int_{Z_{1}}\left(p\left(\mathbf{r} \mid \mathcal{H}_{1}\right)-\lambda p\left(\mathbf{r} \mid \mathcal{H}_{0}\right)\right) d \mathbf{r}+\lambda \alpha
$$


In order to maximize $F$, if the integrand is positive, $p\left(\mathbf{r} \mid \mathcal{H}_{1}\right)-\lambda p\left(\mathbf{r} \mid \mathcal{H}_{0}\right)>0$, we need to assign the result to the region $Z_{1}$. This leads to obtain the following test:

$$
\begin{gathered}
\mathcal{H}_{1} \\
\frac{p\left(\mathbf{r} \mid \mathcal{H}_{1}\right)}{p\left(\mathbf{r} \mid \mathcal{H}_{0}\right)} \underset{\mathcal{H}_{0}}{>} \lambda .
\end{gathered}
$$

The test in (49) is called the Neyman-Pearson test and $\lambda$ should satisfy $P_{F}=\alpha$ [38]. It is important to note that the threshold, $\lambda$, is a function of $P_{F}$.

\subsection{Estimation Theory}

In this section, we briefly introduce the estimation approaches used in the thesis. Depending on whether the prior information for an unknown parameter (the parameter that is desired to be estimated) is obtained or not, we can classify the estimator types into two main categories: Bayesian and Orthodox. MMSE, LMMSE and maximum a posterior estimators are in the Bayesian category that has some knowledge about the unknown parameters. On the other hand, best-linear unbiased, minimum variance and maximum likelihood (ML) estimators are in the Orthodox category that does not require any prior knowledge about the unknown parameters. In this section, we mainly focus on the ML estimator.

Suppose we have an $L \times 1$ unknown parameter vector, $\boldsymbol{\theta}$ and our aim is to estimate $\boldsymbol{\theta}$ from the observation vector, $\mathbf{r} \in \mathbb{C}^{N \times 1}$. The ML estimator maximizes the likelihood function such that:

$$
\hat{\boldsymbol{\theta}}=\underset{\boldsymbol{\theta}}{\operatorname{argmax}} p(\mathbf{r} \mid \boldsymbol{\theta}) \text {. }
$$

The ML estimator has useful properties. For example, the ML estimator is consistent and asymptotically efficient if some regularity conditions are satisfied [36].

The ML estimates may be used for a detection problem. Let us consider again the binary hypothesis testing problem. In both hypotheses, we have a set of unknown parameters. Let $\boldsymbol{\theta}_{0}$ and $\boldsymbol{\theta}_{1}$ be the unknown parameter vectors for $\mathcal{H}_{0}$ and $\mathcal{H}_{1}$, respectively. A detector can be expressed as:

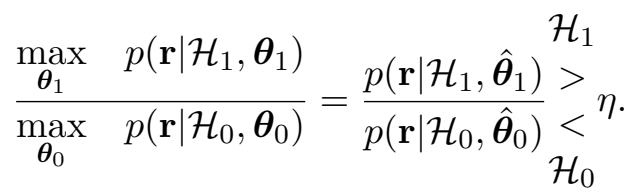


The detector in (51) is called the generalized likelihood ratio test (GLRT) [39]. Although the GLRT is not optimum, in general it gives quite good results.

Now, we analyze the ML estimator for the parameter vector $\boldsymbol{\theta}$, but when the dimension of the parameter vector, $L$, is unknown. Let us assume that $1 \leq L \leq K$. The ML expression can be written:

$$
\hat{L}, \hat{\boldsymbol{\theta}}(\hat{L})=\underset{\boldsymbol{\theta}(L), L}{\operatorname{argmax}} p(\mathbf{r} \mid \boldsymbol{\theta}(L), L) .
$$

Based on (52), the solution of $\hat{L}$ is $K$ because:

$$
\max _{\boldsymbol{\theta}(K)} p(\mathbf{r} \mid \boldsymbol{\theta}(K), K) \geq \max _{\boldsymbol{\theta}(K-1)} p(\mathbf{r} \mid \boldsymbol{\theta}(K-1),(K-1)) \geq \ldots \geq \max _{\boldsymbol{\theta}(1)} p(\mathbf{r} \mid \boldsymbol{\theta}(1), 1) .
$$

Therefore, we conclude that the ML estimator always chooses the most flexible model. In order to prevent this, there exist model order selection rules in the literature.

The first rule that we introduce is the Akaike information criterion. In this rule, the following objective function should be minimized with respect to $L[40]$ :

$$
-2 \log (\mathbf{r} \mid \boldsymbol{\theta}(L))+2 L .
$$

Another rule is the generalized information criterion which minimizes the following [41]:

$$
-2 \log (\mathbf{r} \mid \boldsymbol{\theta}(L))+(1+\rho) L .
$$

where $\rho$ is a parameter which is greater than 1 . If $\rho$ equals to 1 , the generalized information criterion is identical to the Akaike information criterion. The final rule is the Bayesian information criterion (BIC) in which the objective function that should be minimized is [42]:

$$
-2 \log (\mathbf{r} \mid \boldsymbol{\theta}(L))+\log (N) L .
$$

An observation of three information criteria rules is that the first terms in (54), (55) and (56) are the ML expressions and the second terms are penalty functions. Depending on the estimation problem, the performances of these information criteria may be different but in general, the BIC rule gives satisfactory results [41].

\subsection{Basic Optimization Theory and Power Control Schemes}

In this chapter, we briefly introduce some power control schemes. Before this, we explain a general optimization problem. 


\subsubsection{A General Optimization Problem}

A general optimization problem is:

$$
\begin{array}{cl}
\underset{\mathbf{x} \in \mathbb{R}^{N}}{\operatorname{minimize}} & f(\mathbf{x}) \\
\text { subject to } & g_{i}(\mathbf{x}) \leq 0, \quad i=1, \ldots, m, \\
& h_{j}(\mathbf{x})=0, \quad j=1, \ldots, p .
\end{array}
$$

where $f(\mathbf{x})$ is an objective function, $\left\{g_{i}(\mathbf{x})\right\}_{i=1}^{m}$ are inequality constraints and $\left\{h_{j}(\mathbf{x})\right\}_{j=1}^{p}$ are equality constraints. Depending on the convexity or non-convexity of the problem, the solution may be either globally or locally optimum.

The problem in (57) can be rewritten as:

$$
\begin{array}{cl}
\underset{\mathbf{x}, t}{\operatorname{minimize}} & t \\
\text { subject to } & f(\mathbf{x}) \leq t \\
& g_{i}(\mathbf{x}) \leq 0, \quad i=1, \ldots, m, \\
& h_{j}(\mathbf{x})=0, \quad j=1, \ldots, p .
\end{array}
$$

The form in (58) is called the epigraph form and the solutions of (57) and (58) are identical [43].

In Paper A, we encounter geometric programming that is a class of convex optimization problems. If the objective function and the inequality constraints in (57) are posynomials and the equality constraints in (57) are monomials, then the optimization problem in (57) is called geometric programming [43]. The global optimum solution can be found by using numerical techniques.

\subsubsection{Power Control Schemes}

In this section, we explain two power control schemes that are used in Paper A: Max-min fairness and proportional fairness.

Let us consider the uplink scenario. The max-min fairness power control problem can be expressed as:

$$
\begin{array}{lll}
\underset{\eta_{k}}{\operatorname{maximize}} & \min _{k} & \mathrm{SINR}_{k}^{\mathrm{UL}} \\
\text { subject to } & 0 \leq \eta_{k} \leq 1 \quad \forall k .
\end{array}
$$

The aim of max-min fairness is to serve all users equally. 
The proportional fairness problem can be expressed as:

$$
\begin{array}{cl}
\underset{\eta_{k}}{\operatorname{maximize}} & \prod_{k=1}^{K} \mathrm{SINR}_{k}^{\mathrm{UL}} \\
\text { subject to } & 0 \leq \eta_{k} \leq 1 \quad \forall k .
\end{array}
$$

If we consider the high-SNR regime, the proportional fairness approach maximizes the sum SE because:

$$
\log \left(\prod_{k=1}^{K} \operatorname{SINR}_{k}^{\mathrm{UL}}\right)=\sum_{k=1}^{K} \log \left(\operatorname{SINR}_{k}^{\mathrm{UL}}\right) \approx \sum_{k=1}^{K} \log \left(1+\mathrm{SINR}_{k}^{\mathrm{UL}}\right) .
$$




\section{Chapter 5}

\section{Conclusions and Future Work}

In this chapter, we briefly go through what we conclude from this work and some research directions. The detailed results and conclusions can be found in the included papers.

From the results presented in Paper A, although massive MIMO is more robust than the previous generation cellular networks to distributed jammers, it is clear that massive MIMO technology is vulnerable to distributed jamming attacks. It may be an interesting research direction to propose some beamforming techniques as countermeasures for the distributed jammers. However, the challenging part is to estimate the direction-of-arrival of the jamming signals. Another research direction is to investigate different types of jamming signals. Generally, as also in Paper B, the jamming signals are designed based on the Gaussian distribution. The jamming attacks having different distribution than the Gaussian one to massive MIMO may be an interesting research direction.

In Paper B, we propose an algorithm for the mobile GNSS receiver that can detect the multiple spoofers in the environment. The GNSS receiver does not know how many spoofers exists in the environment and which spoofers attack which legitimate signals, i.e., it does not know the attack strategy. By using the algorithm, the GNSS detector can cluster the attack signals and identify the spoofing attack. A limitation of the algorithm is that at least one spoofer should attack more than one legitimate signal. If each spoofer attacks only one legitimate signal, then the algorithm fails. The development of improved algorithms is an open problem. 


\section{Bibliography}

[1] J. G. Andrews, S. Buzzi, W. Choi, S. V. Hanly, A. Lozano, A. C. K. Soong, and J. C. Zhang, "What Will 5G Be?" IEEE Journal on Selected Areas in Communications, vol. 32, no. 6, pp. 1065-1082, 2014.

[2] F. Boccardi, R. W. Heath, A. Lozano, T. L. Marzetta, and P. Popovski, "Five disruptive technology directions for 5G," IEEE Communications Magazine, vol. 52, no. 2, pp. 74-80, Feb. 2014.

[3] E. G. Larsson, O. Edfors, F. Tufvesson, and T. L. Marzetta, "Massive MIMO for next generation wireless systems," IEEE Communications Magazine, vol. 52, no. 2, pp. 186-195, 2014.

[4] J. Zhang, E. Björnson, M. Matthaiou, D. W. K. Ng, H. Yang, and D. J. Love, "Prospective multiple antenna technologies for beyond 5G," IEEE Journal on Selected Areas in Communications, vol. 38, no. 8, pp. 1637-1660, 2020.

[5] D. Bertsekas and J. Tsitsiklis, Introduction to Probability, ser. Athena Scientific books. Athena Scientific, 2002.

[6] T. L. Marzetta, E. G. Larsson, H. Yang, and H. Q. Ngo, Fundamentals of Massive MIMO. Cambridge University Press, 2016.

[7] D. Kapetanovic, G. Zheng, and F. Rusek, "Physical layer security for massive MIMO: An overview on passive eavesdropping and active attacks," IEEE Communications Magazine, vol. 53, no. 6, pp. 21-27, 2015 .

[8] B. Akgun, M. Krunz, and O. Ozan Koyluoglu, "Vulnerabilities of massive MIMO systems to pilot contamination attacks," IEEE Transactions on Information Forensics and Security, vol. 14, no. 5, pp. 1251-1263, 2019.

[9] W. Wang, N. Cheng, K. C. Teh, X. Lin, W. Zhuang, and X. Shen, "On countermeasures of pilot spoofing attack in massive MIMO systems: A double channel training based approach," IEEE Transactions on Vehicular Technology, vol. 68, no. 7, pp. 6697-6708, 2019.

[10] N. Akbar, S. Yan, A. M. Khattak, and N. Yang, "On the pilot contamination attack in multi-cell multiuser massive MIMO networks," IEEE Transactions on Communications, vol. 68, no. 4, pp. 2264-2276, 2020. 
[11] J. K. Tugnait, "Pilot spoofing attack detection and countermeasure," IEEE Transactions on Communications, vol. 66, no. 5, pp. 2093-2106, 2018.

[12] T. T. Do, E. Björnson, E. G. Larsson, and S. M. Razavizadeh, "Jammingresistant receivers for the massive MIMO uplink," IEEE Transactions on Information Forensics and Security, vol. 13, no. 1, pp. 210-223, Jan. 2018.

[13] J. Vinogradova, E. Björnson, and E. G. Larsson, "Jamming Massive MIMO using Massive MIMO: Asymptotic separability results," in 2017 IEEE International Conference on Acoustics, Speech and Signal Processing (ICASSP), 2017, pp. 3454-3458.

[14] H. Wang, K. Huang, and T. A. Tsiftsis, "Multiple antennas secure transmission under pilot spoofing and jamming attack," IEEE Journal on Selected Areas in Communications, vol. 36, no. 4, pp. 860-876, 2018.

[15] E. Kaplan and C. Hegarty, Understanding GPS/GNSS: Principles and Applications, Third Edition, ser. GNSS Technology and Applications Series. Artech House Publishers, 2017.

[16] J. V. Carroll, "Vulnerability assessment of the U.S. transportation infrastructure that relies on the Global Positioning System," Journal of Navigation, vol. 56, no. 2, pp. 185-193, 2003.

[17] A. Jafarnia-Jahromi et al., "GPS vulnerability to spoofing threats and a review of antispoofing techniques," International Journal of Navigation and Observation, vol. 2012, no. 127072, pp. 1-16, July 2012.

[18] K. C. Zeng, S. Liu, Y. Shu, D. Wang, H. Li, Y. Dou, G. Wang, and Y. Yang, "All your GPS are belong to us: Towards stealthy manipulation of road navigation systems," in 27th USENIX Security Symposium (USENIX Security 18). Baltimore, MD: USENIX Association, 2018, pp. 1527-1544. [Online]. Available: https://www.usenix.org/conference/usenixsecurity18/presentation/zeng

[19] E. Axell, E. G. Larsson, and D. Persson, "GNSS spoofing detection using multiple mobile COTS receivers," in 2015 IEEE International Conference on Acoustics, Speech and Signal Processing (ICASSP), 2015, pp. 3192-3196. 
[20] P. Swaszek and R. Hartnett, "Spoof detection using multiple COTS receivers in safety critical applications," in Proceedings of the 26th International Technical Meeting of The Satellite Division of the Institute of Navigation (ION GNSS+ 2013), Sep. 2013, pp. 2921-2930.

[21] A. Kalantari and E. G. Larsson, "Statistical test for GNSS spoofing attack detection by using multiple receivers on a rigid body," EURASIP Journal of Advanced Signal Processing, 2020.

[22] M. Meurer et al., "Robust joint multi-antenna spoofing detection and attitude estimation using direction assisted multiple hypotheses RAIM," Journal of the institute of navigation, pp. 3007-3016, September 2012.

[23] M. L. Psiaki et al., "GNSS spoofing detection using two-antenna differential carrier phase," in Proceedings of the 27th International Technical Meeting of The Satellite Division of the Institute of Navigation (ION GNSS+ 2014), September 2014, pp. 2776-2800.

[24] S. Han, L. Chen, W. Meng, and C. Li, "Improve the Security of GNSS Receivers Through Spoofing Mitigation," IEEE Access, vol. 5, pp. $21057-$ $21069,2017$.

[25] K. Wesson, M. Rothlisberger, and T. Humphreys, "Practical Cryptographic Civil GPS Signal Authentication," NAVIGATION, vol. 59, no. 3, pp. 177-193, 2012.

[26] G. Caparra, S. Sturaro, N. Laurenti, C. Wullems, and R. T. Ioannides, "A Novel Navigation Message Authentication Scheme for GNSS Open Service," in Proceedings of the 29th International Technical Meeting of the Satellite Division of The Institute of Navigation (ION GNSS+ 2016), 2016, pp. $2938-2947$.

[27] T. E. Humphreys, "Detection Strategy for Cryptographic GNSS AntiSpoofing," IEEE Transactions on Aerospace and Electronic Systems, vol. 49, no. 2, pp. 1073-1090, 2013.

[28] D. Tse and P. Viswanath, Fundamentals of wireless communication. Cambridge University Press, 2005.

[29] J. Proakis and M. Salehi, Digital Communications, Fifth Edition. McGraw-Hill Education, 2007.

[30] A. Goldsmith, Wireless Communications. Cambridge University Press, 2005 . 
[31] E. Björnson, J. Hoydis, and L. Sanguinetti, "Massive MIMO networks: Spectral, energy, and hardware efficiency," Foundations and Trends® in Signal Processing, vol. 11, no. 3-4, pp. 154-655, 2017.

[32] R. W. Heath Jr and A. Lozano, Foundations of MIMO communication. Cambridge University Press, 2018.

[33] S. M. Kay, Fundamentals of Statistical Signal Processing: Estimation Theory. Upper Saddle River, NJ, USA: Prentice-Hall, Inc., 1993.

[34] T. Cover and J. Thomas, Elements of Information Theory. Wiley, 2012.

[35] B. Hofmann-Wellenhof, H. Lichtenegger, and J. Collins, Global Positioning System: Theory and Practice. Springer, 1997.

[36] H. Van Trees, K. Bell, and Z. Tian, Detection Estimation and Modulation Theory, Part I: Detection, Estimation, and Filtering Theory, ser. Detection Estimation and Modulation Theory. Wiley, 2013.

[37] J. Nocedal and S. Wright, Numerical Optimization, ser. Springer Series in Operations Research and Financial Engineering. Springer New York, 2006.

[38] S. Kay, Fundamentals of Statistical Signal Processing: Detection theory, ser. Fundamentals of Statistical Signal Processing. Prentice-Hall, 1993.

[39] H. Poor, An Introduction to Signal Detection and Estimation, ser. Springer Texts in Electrical Engineering. Springer New York, 2013.

[40] H. Akaike, "A new look at the statistical model identification," IEEE Transactions on Automatic Control, vol. 19, no. 6, pp. 716-723, 1974.

[41] P. Stoica and Y. Selén, "Model-order selection: a review of information criterion rules," IEEE Signal Process. Mag., vol. 21, no. 4, pp. 36-47, Jul. 2004.

[42] G. Schwarz, "Estimating the dimension of a model," The Annals of Statistics, vol. 6, pp. 461-464, 1978.

[43] S. Boyd and L. Vandenberghe, Convex Optimization. Cambridge University Press, 2004. 


\section{Included Papers}





\section{Papers}

The papers associated with this thesis have been removed for copyright reasons. For more details about these see:

http://urn.kb.se/resolve?urn=urn:nbn:se:liu:diva-172558 



\section{Other Recently Published Theses From \\ The Division of Communication Systems Department of Electrical Engineering (ISY) Linköping University, Sweden}

Giovanni Interdonato, Cell-Free Massive MIMO: Scalability, Signal Processing and Power Control, Linköping Studies in Science and Technology. Dissertations, No. 2090, 2020.

Özgecan Özdogan, Analysis of Cellular and Cell-Free Massive MIMO with Rician Fading, Linköping Studies in Science and Technology. Licentiate Thesis, No. 1870, 2020.

Ema Becirovic, On Massive MIMO for Massive Machine-Type Communications, Linköping Studies in Science and Technology. Licentiate Thesis, No. 1868, 2020.

Daniel Verenzuela, Exploring Alternative Massive MIMO Designs: Superimposed Pilots and Mixed-ADCs, Linköping Studies in Science and Technology. Dissertations, No. 2041, 2020.

Trịnh Văn Chiến, Spatial Resource Allocation in Massive MIMO Communication: From Cellular to Cell-Free, Linköping Studies in Science and Technology. Dissertations, No. 2036, 2020.

Amin Ghazanfari, Power Control for Multi-Cell Massive MIMO, Linköping Studies in Science and Technology. Licentiate Thesis, No. 1852, 2019.

Marcus Karlsson, Blind Massive MIMO Base Stations: Downlink Transmission and Jamming, Linköping Studies in Science and Technology. Dissertations, No. 1950, 2018.

Victor Hei Cheng, Optimizing Massive MIMO: Precoder Design and Power Allocation, Linköping Studies in Science and Technology. Dissertations, No. 1929, 2018.

Christopher Mollén, High-End Performance with Low-End Hardware: Analysis of Massive MIMO Base Station Transceivers, Linköping Studies in Science and Technology. Dissertations, No. 1896, 2017.

Antonios Pitarokoilis, Phase Noise and Wideband Transmission in Massive MIMO, Linköping Studies in Science and Technology. Dissertations, No. 1756, 2016.

Anu Kalidas M. Pillai, Signal Reconstruction Algorithms for Time-Interleaved ADCs, Linköping Studies in Science and Technology. Dissertations, No. 1672, 2015.

Ngô Quốc Hiển, Massive MIMO: Fundamentals and System Designs, Linköping Studies in Science and Technology. Dissertations, No. 1642, 2015. 


\title{
FACULTY OF SCIENCE AND ENGINEERING
}

\author{
Linköping Studies in Science and Technology, \\ Licentiate Thesis No. 1899, 2021 \\ Division of Communication Systems \\ Department of Electrical Engineering \\ Linköping University \\ SE-581 83 Linköping, Sweden
}

www.liu.se 